\title{
ON SOME NEW THEOREMS IN THE THEORY OF DIOPHANTINE APPROXIMATIONS
}

By

VERA T. SÓS (Budapest) and P. TURÁN (Budapest), member of the Academy

1. In a recent book $^{1}$ one of us based a series of applications on the following three theorems.

I. If

$$
\left|z_{1}\right| \geqq\left|z_{2}\right| \geqq \cdots \geqq\left|z_{i}\right|
$$

$m$ is a non-negative integer, the $b_{j}$ 's are arbitrary complex numbers, there is then an integer $y_{\perp}$ such that

and

$$
m+1 \leqq \nu_{1} \leqq m+n
$$

$$
\left|b_{1} z_{1}^{p_{1}}+b_{2} z_{2}^{n_{1}}+\cdots+b_{n} z_{n}^{\nu_{1}}\right| \geqq\left|z_{n}\right|^{\nu_{1}}\left(\frac{n}{2 e(m+n)}\right)^{n}\left|b_{1}+\cdots+b_{n}\right| \text {. }
$$

II. With the above notations there is an integer $v_{2}$ such that

and

$$
m+1 \leqq \nu_{2} \leqq m+n
$$

$$
\left|b_{1} z_{1}^{\nu_{2}}+b_{2} z_{2}^{\nu_{2}}+\cdots+b_{n} z_{z^{\prime}}^{\nu_{2}}\right| \geqq\left|z_{1}^{\mid \gamma_{2}}\left(\frac{n}{24 e^{2}(m+2 n)}\right)^{n} \min _{j=1, \ldots, n}\right| b_{1}+\cdots+b_{j} \mid \cdot
$$

III. With the above notations and

$$
\omega(z)=\prod_{r=1}^{n}\left(z-z_{r}\right)
$$

there is an integer $\nu_{3}$ such that

and

$$
m+1 \leqq v_{3} \leqq m+n
$$

$$
\begin{aligned}
& \left|b_{1} z_{1}^{\nu_{3}}+b_{z} z_{2}^{\gamma_{3}}+\cdots+b_{l} z_{13}^{\nu_{3}}\right| \geqq \\
& \geqq\left(\sum_{j=1}^{i n}\left|b_{j}\right|\left|z_{j}\right|^{\nu_{3}}\right) \cdot \prod_{l=1}^{n} \frac{1}{1+\left|z_{l}\right|} \cdot \max _{k=0,1, \ldots,(n-1)} \sum_{l=1}^{n} \frac{1}{\frac{\left|z_{l}\right|^{k}}{\left|\omega^{\prime}\left(z_{l}\right)\right|\left(1+\left|z_{l}\right|\right)}} .
\end{aligned}
$$

In mentioned book ${ }^{1}$ III was discussed mainly as a matter of orientation and had only one application in the investigation of integral functions of type

$$
\sum_{\nu=1}^{n} a_{\nu} F\left(c_{\nu} z\right)
$$

1. P. Tưán, Eine neue Methode in der Analysis und deren Anwendungen (Budapest, 1953), Akadémiai Kiadó. 
where $F(z)$ is an integral function. Since that time the second of us found some more applications of it. To show the essence of these three Theorems I, II, III we call the quantities

$$
\left.\sum_{j=1}^{n}\left|b_{j}\right| z_{j}\right|^{r^{\prime}}, \quad\left|z_{n}\right|^{p^{\prime}}, \quad\left|z_{1}\right|^{\prime}
$$

$N_{i}\left(w^{\prime}, f\right)$ norms of $f(x)=\sum_{j=1}^{n} b_{j} z_{j}^{\prime \prime}(l=1,2,3)$. Then the Theorems I, II and III can be expressed by saying that, for a suitable integer $y,\left|\sum_{j=1}^{n} b_{j} z_{j}^{\prime}\right|$ is estimated from below by the $N_{l}(v, f)$ norms (1.1) so, that the lower estimation of their quotient should be independent

a) of the $z_{j}$-values

(1. 2) or

b) of the $b_{j}$ coefficients.

Theorems I and II are of a)-type, Theorem III is of b)-type. This formulation of the theory is more symmetrical than that given in ${ }^{1}$, where only problems of a)-type were systematically treated. In connection with an application $^{2}$ the necessity of dual theorems emerged where non-trivial upper estimations of

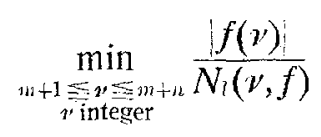

are needed even at the rate of simple geometrical restrictions on the $z_{j}$ 's. In ${ }^{1}$ one can find detailed motivation, on which way Theorems I, II and III can be considered as generalisations of KRONECKER's and DiRICHLET's classical theorems in the theory of diophantine approximations.

2. In ${ }^{1}$ the emphasis was laid upon the applicability of these theorems and no care was taken to best-possible inequalities, though these have a significance for some applications, too. One can show this e.g. on the estimation " of $N(\alpha, T)$, the number of zeros of $\zeta(s)(s=\sigma+i t)$ in the parallelogram $\sigma \geqq e, 0<t \leqq T$

$$
N(\alpha, T)=O\left(T^{2(1-\alpha)+(1-\alpha)^{1,14}}\right)
$$

which is uniformly valid for

$$
1-\delta \leqq \varkappa \leqq 1
$$

with a (small) numerical positive $\delta$. This constitutes the best-known estima$145-163$.

2 P. Turais, On Lindelöf's conjecture, Acta Math. Acad. Sci. Hung., 5 (1954), pp.

${ }^{3}$ This is an unpublished sharpening of Theorem XXXVIll of 1. 
tion in this range today. The proof of this is largely based on the case

$$
b_{1}=b_{2}=\cdots=b_{n}=1
$$

of Theorem II; thereby the decrease of the numerical factor $24 e^{2}$, since $n$ is "large" in this case, would result an increase of $\delta$ in (2.2) which in turn would result a decrease of the smallest known $\Theta$ with the property

$$
p_{n+1}-p_{n}=O\left(p_{n}^{\Theta}\right)
$$

where $p_{n}$ denotes the $n^{\text {th }}$ prime. The main aim of this paper is to review the a)-type results in the first part of ${ }^{1}$ from this point of view, in particular in the case (2.3), and to study certain $\left(z_{1}, z_{2}, \ldots, z_{n}\right)$-systems, which will play a role in these questions. We suppose without loss of generality

$$
z_{n}=1, \quad\left|z_{j}\right| \geqq 1 \quad(j=1, \ldots, n)
$$

in $\mathbf{I}$ and

$$
z_{1}=1, \quad\left|z_{j}\right| \leqq 1 \quad(j=1, \ldots, n)
$$

in II. In the case (2.5) we ask for the "smallest" numerical positive value $A_{1}$, for which

$$
\max _{\substack{m+1 \\ \nu \text { integer }}}\left|z_{1}^{\nu}+z_{2}^{\nu}+\cdots+z_{n}^{\nu}\right| \geqq\left(\frac{n}{A_{1}(m+n)}\right)^{n}
$$

holds for an arbitrary non-negatíve integer $m$ and positive integer $n$. We shall show in 6-9 the following

THEOREM. We have for the $A_{1}$ defined in (2.6) the inequality

$$
1,321<A_{1}<2 e^{1+\frac{4}{e}}(<24) \text {. }
$$

The gap is still large but the upper bound is much better than the previous $24 e^{2} \sim 177$. That $A_{1}>1,1$, can already be shown taking

$$
m=0, \quad n=2, \quad z_{1}=1, \quad z_{2}=\frac{1}{2} e^{\frac{2 \pi i}{3}}
$$

in this case we have

i. e.

$$
\left|z_{1}+z_{2}\right|=\frac{\sqrt{3}}{2}, \quad\left|z_{1}^{2}+z_{2}^{2}\right|=\frac{\sqrt{13}}{4},
$$

$$
\frac{\sqrt{13}}{4} \geqq \frac{1}{A_{1}^{2}}, \quad A_{1} \geqq \frac{2}{\sqrt[4]{13}}>1,1 .
$$

An interesting feature of the proof of the upper bound is the avoiding of the use of H. CARTAN's theorem and replacing it by a lemma of CHEBYSEv-type. The new proof furnishes mutatis mutandis the following improvement of II. 
Under the conditions of II we have (2. 7)

$\left|b_{1} z_{1}^{\nu_{2}}+\cdots+b_{n} z_{n}^{\nu_{*}}\right| \geqq\left|z_{1}\right|^{\nu_{2}}\left(\frac{n}{2 e^{1+\frac{4}{e}}(m+n)}\right)^{n} \cdot \min _{j=1, \ldots, n}\left|b_{1}+\cdots+b_{j}\right|$

It will be sufficient to indicate only the changes, the proof of (2.7) needs compared to that of the theorem.

3. A further refinement of the upper estimation in the Theorem would be given if the constant $2 e$ in I could be diminished, even only in the special case $b_{1}=\cdots=b_{n}=1$. Asking for the "smallest" numerical positive value $A_{2}$, for which in the case (2.4)

$$
\max _{\substack{m+1 \\ \text { integer }}}\left|z_{1}^{\nu}+z_{2}^{\nu}+\cdots+z_{n}^{\nu}\right| \geqq\left(\frac{n}{A_{2}(m+n)}\right)^{n}
$$

holds for any positive integer $n$ and non-negative integer $m$, nothing better than

$$
1 \leqq A_{2} \leqq 2 e
$$

can be asserted at the present. Something better can be said on the "smallest" numerical positive value $A_{3}$ for which in the case (2.4)

$$
\max _{\substack{m+1 \leq \nu \leq 4 \\ \nu \text { integer }}}\left|b_{1} z_{1}^{v}+\cdots+b_{n} z_{n}^{v}\right| \geqq\left(\frac{n}{A_{3}(m+n)}\right)^{n}\left|b_{1}+\cdots+b_{n}\right|
$$

holds for any complex $b_{v}$ 's, positive integer $n$ and non-negative integer $m$. We shall show in $\mathbf{1 0}$ that

$$
1,27 \sim \frac{4}{\pi} \leqq A_{3} \leqq 2 e \sim 5,44 .
$$

4. The lower limitation of $A_{1}$ in the Theorem will be proved in 9 by refining an idea of $\mathrm{P}$. ERDós, i. e. considering $\left(z_{1}, z_{2}, \ldots, z_{n}\right)$-systems with the property

$$
s_{2}=s_{3}=\cdots=s_{n-1}=0, \quad z_{1}=1
$$

where $s_{v}$ stands for $z_{1}^{v}+z_{2}^{v}+\cdots+z_{n}^{v}$. This suggests for the sake of counter examples the usefulness of the study of all $\left(z_{1}, \ldots, z_{n}\right)$-systems with the property

$$
s_{2}=s_{3}=\cdots=s_{n-1}=s_{n}=0,
$$

or more generally with a prescribed non-negative integer $m$ the determination of all those with

$$
s_{m+1}=s_{m+2}=\cdots=s_{m+n-1}=0 .
$$

We mention another reason why (4.2) is interesting. The whole theory emerged from the necessity to diminish the interval for $\nu_{j}$ in I, II and III as much as 
possible; the question arises now, whether or not for some integer values $m$ there is a non-trivial lower limitation for

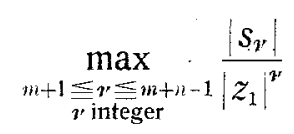

or for

$$
\max _{m+1 \leq v \leq m+n-1} \frac{\left|s_{y}\right|}{\left|z_{n}\right|^{\nu^{\prime}}} .
$$

That such a reduction of the interval for $v$ is generally impossible, is trivial, since in the case $m=0 \bmod n$ we have for the $n^{\text {th }}$ roots of unity

$$
s_{m+1}=s_{m+2}=\cdots=s_{m+n-1}=0 .
$$

Probably the same holds for all non-negative integer values $m$. As the NewtonGirard formulae show at once, for $m=0$ all the $\left(z_{1}, \ldots, z_{n}\right)$-systems with

$$
s_{1}=s_{2}=\cdots=s_{n-1}=0
$$

are given by the zeros of an equation

$$
z^{n}+a=0 \quad(a \text { arbitrary complex }) .
$$

We can determine all systems satisfying (4.2) with $m=1$ and $m=2$. For $m=1$ we assert that all $\left(z_{1}, z_{2}, \ldots, z_{i n}\right)$-systems with the property

$$
s_{2}=s_{3}=\cdots=s_{n}=0 \text {. }
$$

are formed by the zeros of an equation

$$
\varphi_{n}(z, a)=z^{n}+\frac{a}{1 !} z^{n-1}+\cdots+\frac{a^{n}}{n !}=0
$$

(a arbitrary complex).

An asymptotical determination of these systems for fixed $a$ and $n \rightarrow \infty$ follows at once from SzEgö's ${ }^{4}$ results. For $m=2$ we shall see in 11 that all the $\left(z_{1}, z_{2}, \ldots, z_{n}\right)$-systems with

$$
s_{3}=s_{4}=\cdots=s_{n}=s_{n+1}=0
$$

are formed by the zeros of an equation.

$$
f_{n}(z, a, \lambda)=z^{n}+\frac{H_{1}(\lambda)}{1 !} a z^{n-1}+\cdots+\frac{H_{n}(\lambda)}{n !} a^{n}=0,
$$

where $H_{\nu}(y)$ stands for the $v^{\text {th }}$ Hermite polynomial defined by

$$
H_{\nu}(y)=(-1)^{v} e^{y^{2}} \frac{d^{v}}{d y^{\nu}} e^{-y^{2}},
$$

$\lambda$ denotes any zero of $H_{n+1}(y)=0$ and $a$ is an arbitrary complex number. An asymptotical determination of the zeros of $f_{n}(z, a, \lambda)$ is not known. The

* G. Szegö, Uber eine Eigenschaft der Exponentialreihe, Sitzungsber. der Berl. Math. Ges., 23 (1924), pp. 50-63. 
result (4.5)-(4.6) can be easily ${ }^{5}$ proved by Newton-Girard formulae so we omit the details. It is interesting to remark a characteristic difference between the cases $m=1$ and $m=2$. All solutions of $(4,5)$ can be derived from the single equation

$$
1+\frac{z}{1 !}+\frac{z^{2}}{2 !}+\cdots+\frac{z^{n}}{n !}=0
$$

all solutions of (4.7), however, can be derived from the $(n+1)$ equations

$$
\sum_{\nu=0}^{n} \frac{H_{n}(\lambda)}{v !} z^{\prime}=0 \quad \text { where } \quad H_{n+1}(\lambda)=0 .
$$

5. Owing to the applicability to the approximative solution of algebraic equations it is of interest to study II in the special case

$$
m=0, \quad b_{1}=b_{2}=\cdots=b_{n}=1 .
$$

In this case we have to determine

$$
M_{n}=\min _{\substack{\left|z_{j}\right| \leqq 1 \\ j=1, \ldots, n}} \max _{p=1, \ldots, n}\left|1+z_{2}^{p}+\cdots+z_{n}^{\nu}\right| .
$$

In ${ }^{1}$ we have shown

$$
M_{i} \geqq \frac{\log 2}{\frac{1}{1}+\frac{1}{2}+\cdots+\frac{1}{n}} ;
$$

according to a written communication of DE BRUIN this can be replaced by

$$
M_{n}>c \frac{\log \log n}{\log n} \quad(c \text { numerical positive constant), }
$$

what is for large $n$ somewhat better. This makes still more probable the conjecture that $M_{n} \geqq d$, independently of $n$. As to the upper estimation of $M_{n}$ Mr. Hyltén-Cavallius showed $M_{2}=\frac{\sqrt{5}-1}{\sqrt{2}} \sim 0,86$ and found by considering $z_{2}=0,1295+i 0,7063, z_{3}=-0,5128+i 0,1508$ the estimation

$$
M_{8}<0,831 \text {. }
$$

6. Before turning to the proof of the Theorem we need the following simple

LEMMA. Let be $0 \leqq \delta \leqq 1$ and

$$
f(z)=z^{n}+\cdots=\prod_{\eta=1}^{n}\left(z-z_{\eta}\right) .
$$

5 Another very elegant verification is due to E. Egerváry. Using Euler's formulae $\sum_{j=1}^{n} \frac{z_{j}^{y}}{\omega^{\prime}\left(z_{j}\right)}=0(v=0,1, \ldots, n-2)$ valid for any polynomial $o(z)$ with simple $z_{j}$-zeros of degree $n$ and using $\varphi_{n}^{\prime}\left(z_{j}, a\right)=\left(n ! z_{j}^{2}\right)^{-1} a^{n+1}$ the result follows at once. 
Then, there is a circle $|z|=r_{0}$ with $\delta \leqq r_{0} \leqq 1$ such that on the whole periphery

$$
|f(z)| \geqq 2\left(\frac{1-\delta}{4}\right)^{n}
$$

For the proof we introduce

$$
f^{*}(z)=\prod_{\nu=1}^{n}\left(z-\left|z_{\nu}\right|\right)
$$

Owing to the classical theorem of CHËBYSEv there is a $\xi$ with $\delta \leqq \xi \leqq 1$ and

$$
\left|f^{*}(\xi)\right| \geqq 2\left(\frac{1-\delta}{4}\right)^{n}
$$

But on the circle $|z|=\xi$ we have

$$
\left|z-z_{j}\right| \geqq|| z|-| z_{j}||=|\xi-| z_{j}|| \quad(j=1,2, \ldots, n),
$$

i. e. multiplying, further using (6.2) and (6.3) we get

$$
|f(z)| \geqq\left|f^{*}(\xi)\right| \geqq 2\left(\frac{1-\delta}{4}\right)^{n} .
$$

Hence the above $\xi$ can be chosen as $r_{0}$. Q. e. d.

7. Next we turn to the proof of our Theorem. We may suppose that $n \geqq 2$. Let be, with our numbers $z_{j}$,

$$
\omega(z)=\prod_{j=1}^{n}\left(z-z_{j}\right)
$$

and let $\delta$ be a positive number $0 \leqq \delta \leqq 1$ to be determined later. To this $\omega(z)$ and $\delta$ there is, according to the Lemma, an $r_{0}$ with $\delta \leqq r_{0} \leqq 1$ and such that for $|z|=r_{0}$

$$
|\omega(z)| \geqq 2\left(\frac{1-\delta}{4}\right)^{n}
$$

Since each factor $\left|z-z_{j}\right|$ is at most 2 , we have from (7.2) for $|z|=r_{0}$ and for any choice of the indices $(1 \leqq) i_{1}<i_{2}<\cdots<i_{k} \leqq n(1 \leqq k \leqq n)$

$$
\prod_{v=1}^{k}\left|z-z_{i_{v}}\right| \geqq 2\left(\frac{1-\delta}{4}\right)^{n} \frac{1}{2^{n-k}}=2\left(\frac{1-\delta}{8}\right)^{n} 2^{z^{k}} .
$$

We investigate two cases.

Case a) All $z_{j}$ 's are absolutely $\geqq r_{0}$. Then, owing to $\mathbf{I}$, there is an integer $\nu_{1}$ with $m+1 \leqq \nu_{1} \leqq m+n$ and

$$
\left|z_{1}^{\nu_{1}}+z_{2}^{p_{1}}+\cdots+z_{n}^{\nu_{1}}\right| \geqq r_{0}^{\nu_{1}}\left(\frac{n}{2 e(m+n)}\right)^{n} \geqq \delta^{m+n}\left(\frac{n}{2 e(m+n)}\right)^{n} .
$$

Case b) There is an index $l$ with $1 \leqq l<n$ and

$$
1=\left|z_{1}\right| \geqq\left|z_{2}\right| \geqq \cdots \geqq\left|z_{l}\right|>r_{0}>\left|z_{l+1}\right| \geqq \cdots \geqq\left|z_{n}\right| \text {. }
$$


In the treatment of this case we shall suppose first that the $z_{j}$ 's are all different.

8. Let first be

$$
f_{1}(z)=\prod_{j=l+1}^{n}\left(z-z_{j}\right)=\sum_{j=0}^{n-l} c_{j}^{(1)} z^{n-l-j}, \quad c_{0}^{(1)}=1 .
$$

We have obviously

$$
\left|c_{j}^{(1)}\right|=\left|\sum_{l+1 \leqq i_{1}<i<i_{j} \leqq n} z_{i_{1}} z_{i_{2}} \cdots z_{i_{j}}\right| \leqq\left(\begin{array}{c}
n-l \\
j
\end{array}\right) .
$$

Next let $f_{2}(z)$ be that polynomial of degree $\leqq l-1$, which assumes for $z=z_{1}, z_{2}, \ldots, z_{l}$ the values

$$
\frac{1}{z_{1}^{n+1} f_{1}\left(z_{1}\right)}, \frac{1}{z_{2}^{n+1} f_{1}\left(z_{2}\right)}, \ldots, \frac{1}{z_{l}^{m+1} f_{1}\left(z_{l}\right)},
$$

respectively. If $l=1$, then

$$
f_{2}(z) \equiv \frac{1}{z_{1}^{m+1} f_{1}\left(z_{3}\right)} \equiv c_{0}^{(2)} ;
$$

if $1<l<n$, then we can represent $f_{2}(z)$ as Newton-interpolatorical polynomial $f_{2}(z)=c_{0}^{(2)}+c_{1}^{(2)}\left(z-z_{1}\right)+c_{2}^{(2)}\left(z-z_{1}\right)\left(z-z_{2}\right)+\cdots+c_{l-1}^{(2)}\left(z-z_{1}\right)\left(z-z_{2}\right) \cdots\left(z-z_{l-1}\right)$.

Since the function $\frac{1}{z^{m+1} f_{1}(z)}$ is regular for $|z|>r_{0}$ and vanishes for $z=\infty$, we have according to NöRLUND's representation

$$
\begin{gathered}
c_{j}^{(2)}=\frac{1}{2 \pi i} \int_{|x|=x_{0}} \frac{d w}{w^{m+1} f_{1}(w)\left(w-z_{1}\right)\left(w-z_{3}\right) \cdots\left(w-z_{j+1}\right)} \\
(j=0,1, \ldots,(l-1)) .
\end{gathered}
$$

But $f_{1}(w)\left(w-z_{1}\right) \cdots\left(w-z_{j+1}\right)$ is of type (7.3) with $k=n-l+j+1$ and thus owing to (7.3) we have for $|w|=r_{0}$

$$
\left|f_{1}(w)\left(w-z_{1}\right)\left(w-z_{2}\right) \cdots\left(w-z_{j+1}\right)\right| \geqq 2\left(\frac{1-\delta}{8}\right)^{n} 2^{n-1+j+1}=2\left(\frac{1-\delta}{4}\right)^{n} \frac{1}{2^{1-j-1}},
$$

i. e. from (8.3)

$$
\left|c_{j}^{(2)}\right| \leqq \frac{1}{2 r_{0}^{m}}\left(\frac{4}{1-\delta}\right)^{n} 2^{1-j-1} \leqq \frac{1}{2 \delta^{3 n}}\left(\frac{4}{1-\delta}\right)^{n} 2^{i-j-1} .
$$

But we need also $f_{2}(z)$ in the form

$$
f_{2}(z)=\sum_{j=0}^{l-1} c_{j}^{(i)} z^{j}
$$

and we have to estimate the coefficients $c_{j}^{(3)}$. We have

$$
c_{l-1}^{(3)}=\boldsymbol{c}_{-1}^{(9)}
$$


and if $l>1$, for $j=0,1, \ldots,(l-2)$

i. e.

$$
\begin{gathered}
c_{j}^{(3)}=c_{j}^{(2)}-c_{j+1}^{(2)} \sum_{1 \leqq r_{1} \leqq j+1} z_{r_{1}}+c_{j+2}^{(2)} \sum_{1 \leqq r_{1}<r_{2} \leqq j+2} z_{r_{1}} z_{r_{2}}-\cdots+ \\
+(-1)^{l-j-1} c_{l-1}^{(2)} \sum_{1 \leqq x_{1}<r_{2}<\ldots<r_{l-j-1} \leqq l-1} z_{r_{1}} z_{r_{2}} \cdots z_{r_{l-j \cdot 1}},
\end{gathered}
$$

$$
\left|c_{j}^{(3)}\right| \leqq\left|c_{j}^{(2)}\right|+\left|c_{j+1}^{(2)}\right|\left(\begin{array}{c}
j+1 \\
1
\end{array}\right)+\left|c_{j+2}^{(2)}\right|\left(\begin{array}{c}
j+2 \\
2
\end{array}\right)+\cdots+\left|c_{l-1}^{(2)}\right|\left(\begin{array}{c}
l-1 \\
l-j-1
\end{array}\right)
$$

Thus from $(8.4)$

$$
\begin{gathered}
\left|c_{j}^{(3)}\right| \leqq \frac{2^{l-j-1}}{2 \delta^{m}}\left(\frac{4}{1-\delta}\right)^{n}\left\{1+\left(\begin{array}{c}
j+1 \\
1
\end{array}\right) \frac{1}{2}+\left(\begin{array}{c}
j+2 \\
2
\end{array}\right) \frac{1}{2^{2}}+\cdots+\left(\begin{array}{c}
l-1 \\
l-1-j
\end{array}\right) \frac{1}{2^{l-j-1}}\right\}< \\
(8.5) \quad \frac{2^{l-j-1}}{2 \delta^{m}}\left(\frac{4}{1-\delta}\right)^{n} \sum_{d=0}^{\infty}\left(\begin{array}{c}
j+d \\
d
\end{array}\right) \frac{1}{2^{d}}= \\
=\frac{2^{l-j-1}}{2 \delta^{m}}\left(\frac{4}{1-\delta}\right)^{n} \sum_{d=0}^{\infty}(-1)^{d}(-j-1) \frac{1}{d}=\frac{2^{l-1}}{\delta^{m}}\left(\frac{4}{1-\delta}\right)^{n} .
\end{gathered}
$$

Let finally be

$$
f_{3}(z)=z^{m+1} f_{1}(z) f_{2}(z)=\sum_{j=m+1}^{m+n} c_{j}^{(4)} z^{j}
$$

It follows from the definition of $f_{1}(z)$ and $f_{2}(z)$ that

$$
f_{3}\left(z_{1}\right)=f_{3}\left(z_{2}\right)=\cdots=f_{3}\left(z_{l}\right)=1, f_{3}\left(z_{l+1}\right)=\cdots=f_{3}\left(z_{n}\right)=0 \text {. }
$$

Replacing $z$ in (8.6) by $z_{j}$ and ${ }^{6}$ summing for $j=1,2, \ldots, n$ we get, using (8: 7) and writing

$$
s_{v}=z_{1}^{\nu}+z_{2}^{\nu}+\cdots+z_{n}^{\nu}
$$

the identity

i. e.

$$
\left|\sum_{\nu=m+1}^{m+n} c_{\nu}^{(4)} s_{\nu}\right|=\sum_{\nu=m+1}^{m+n} c_{\nu}^{(4)} s_{\nu}=l
$$

$$
l \leqq \max _{\substack{m+1 \leqq \nu \leqq m+n \\ \nu \text { integer }}}\left|s_{\nu}\right|\left(\sum_{j=m+1}^{m+n n}\left|c_{j}^{(4)}\right|\right)
$$

But from (8.6) we have

i. e.

$$
c_{j}^{(4)}=\sum_{n-l-j_{1}+j_{2}=j-m-1} c_{j_{1}}^{(1)} c_{j_{2}}^{(3)}
$$

$$
\sum_{j=m+1}^{m+n}\left|c_{j}^{(4)}\right| \leqq\left(\sum_{j=0}^{n-l}\left|c_{j_{1}}^{(1)}\right|\right)\left(\sum_{j_{2}=0}^{l-1}\left|c_{j_{2}}^{(3)}\right|\right)
$$

${ }^{6}$ If we want to prove (2.7) instead of the upper estimation of the Theorem, we have also to multiply by $b_{j}$ and then to sum for $j$. 
thus, using (8.2) and (8.5), we get

$$
\sum_{j=m+1}^{m+n}\left|c_{j}^{(4)}\right| \leqq 2^{n-l} l \frac{2^{l-1}}{\delta^{m}}\left(\frac{4}{1-\delta}\right)^{n}=\frac{l}{2 \delta^{m}}\left(\frac{8}{1-\delta}\right)^{n} .
$$

Then we have from (8.9)

$$
\max _{\substack{m+1 \\ \nu \text { integer }}}\left|s_{\nu}\right| \geqq 2 \delta^{m}\left(\frac{1-\delta}{8}\right)^{n} .
$$

Now we choose $\delta$ so, that

i. e.

$$
\delta^{m+n}\left(\frac{n}{2 e(m+n)}\right)^{n}=\delta^{m}\left(\frac{1-\delta}{8}\right)^{n}
$$

$$
\delta=\frac{1}{1+\frac{4}{e} \frac{n}{m+n}} \equiv \delta_{0}
$$

then from (7.4) and (8.10) we got

But

$$
\max _{\gamma=m+1, m+2, \ldots, m+n}\left|z_{1}^{\gamma}+z_{2}^{\gamma}+\cdots+z_{n}^{v}\right| \geqq 2 \delta_{0}^{m+n}\left(\frac{n}{2 e(m+n)}\right)^{n} .
$$

$$
\left(1+\frac{4 n}{e(m+n)}\right)^{m+n} \leqq e^{\frac{4 n}{e}}, \text { i. e. } \delta_{0}^{m+n} \geqq e^{-\frac{4 n}{e}}
$$

whence

$$
\max _{\nu=m+1, m+2, \ldots, m+n}\left|z_{1}^{\nu}+\cdots+z_{n}^{\nu}\right| \geqq 2\left(\frac{n}{2 e^{1+\frac{4}{e}}(m+n)}\right)^{n},
$$

i. e. our Theorem is for different $z_{j}$ 's proved. We can get rid of the restriction $z_{\mu} \neq z_{\nu}(\mu \neq \nu)$ exactly on the same way as in the previous proof in ${ }^{1}$ and we do not detail it.

9. Now we turn to the lower estimation in the Theorem. Let 9 be a positive constant, less than 1 and to be determined later, let $z_{1}=1$ and $z_{2}, z_{3}, \ldots, z_{n}$ be determined by the conditions (with the notation (8.8))

$$
s_{1}=9 n, s_{2}=s_{3}=\cdots=s_{n-1}=0 \text {. }
$$

It is well known that these conditions determine uniquely the numbers $z_{j}(j=2,3, \ldots, n)$; let us denote them by $\zeta_{v}\left(\zeta_{1}=1, v=2,3, \ldots, n\right)$. Let further be

$$
f(z)=z^{n}+a_{1} z^{n-1}+\cdots+a_{n}
$$

the polynomial with the zeros $\zeta_{n}(\nu=1, \ldots, n)$. Then the Newton-Girard formulae give successively

$$
a_{1}=-s_{1}, a_{2}=\frac{s_{1}^{2}}{2 !}, a_{3}=-\frac{s_{1}^{3}}{3 !}, \ldots, a_{n-1}=(-1)^{n-1} \frac{s_{1}^{n-1}}{(n-1) !} .
$$


Since $\zeta_{1}=1$ is a zero of $f(z)$, we have

$$
a_{n}=-\left(1+a_{1}+\cdots+a_{n-1}\right)=-\left(1-\frac{s_{1}}{1 !}+\frac{s_{1}^{2}}{2 !}-\cdots+(-1)^{n-1} \frac{s_{1}^{n-1}}{(n-1) !}\right)=
$$

$$
=-e^{-s_{1}}+\sum_{j=1}^{\infty}(-1)^{j} \frac{1}{j !} s_{1}^{j} \text {. }
$$

Further, we have from Newton-Girard formulae

$$
\begin{gathered}
s_{n}=-a_{n-1} s_{1}-n a_{n}=(-1)^{n} \frac{s_{1}^{n}}{(n-1) !}+n e^{-s_{1}}-\sum_{j=n}^{\infty} n(-1)^{j} \frac{s_{1}^{j}}{j !}= \\
=n\left(e^{-s_{1}}-\sum_{j=n+1}^{\infty}(-1)^{j} \frac{s_{1}^{j}}{j !}\right)=n \sum_{j=0}^{n}(-1)^{j} \frac{s_{1}^{j}}{j !}
\end{gathered}
$$

and from (9.3) and (9.4)

$$
\begin{gathered}
s_{n+1}=-a_{1} s_{n}-a_{n} s_{1}=s_{1}\left(s_{n}-a_{n}\right)= \\
=s_{1}\left\{(n+1) e^{-s_{1}}+(-1)^{n+1} \frac{s_{1}^{n}}{n !}-(n+1) \sum_{j=n+1}^{\infty}(-1)^{j} \frac{s_{1}^{j}}{j !}\right\} .
\end{gathered}
$$

Since $0<\boldsymbol{9}<1$, the terms $\frac{s_{1}^{j}}{j !}$ decrease monotonically if $j \geqq n$, it follows

$$
\left|\sum_{j=n+1}^{\infty}(-1)^{j+1} \frac{s_{1}^{j}}{j !}\right|<\frac{s_{1}^{n+1}}{(n+1) !},
$$

i. e. from (9.4) and (9.5)

$$
\left|s_{n}\right| \leqq n\left(e^{-\vartheta^{\vartheta}}+\frac{(\vartheta n)^{n+1}}{(n+1) !}\right), \quad\left|s_{n+1}\right| \leqq \vartheta n\left\{(n+1) e^{-\vartheta_{n}}+\frac{(\vartheta n)^{n z}}{n !}+\frac{(\vartheta n)^{n+1}}{n !}\right\} \text {. }
$$

Since $k !>\left(\frac{k}{e}\right)^{k} e$, we get for $n>10$

$$
\left|s_{n}\right| \leqq n\left\{e^{-\vartheta_{n}}+\frac{1}{e}(e \vartheta)^{n+1}\right\}, \quad\left|s_{n+1}\right| \leqq(n+1)^{2}\left(e^{-\vartheta_{n}}+4(e \vartheta)^{n}\right)
$$

whence with $m=1$

$$
\max _{n+1 \leqq \nu \leqq}\left|s_{\nu}\right|<4(n+1)^{2}\left\{e^{-y_{n}}+(e \vartheta)^{n}\right\}
$$

If $\vartheta$ is the (only) positive zero $c_{0}$ of the transcendental equation

$$
x=e^{-x-1} \text {, }
$$

then we have $0,2784 \leqq \vartheta \leqq 0,2785$ and for our $\zeta_{\nu}$ 's

$$
\max _{\substack{i \leq \nu \leq n+1 \\ \nu \text { integer }}}\left|s_{\nu}\right| \leqq 8(n+1)^{2} e^{-a_{0} n} \text {. }
$$

It may occur that (2.5) is not fulfilled by the $\zeta_{\nu}$-system, i. e. some of them are absolutely $\geqq 1$. If so, we can construct simply by contraction a $\zeta_{v}^{*}$-system 
satisfying (2.5) and for which (9.7) holds a fortiori. Thus for an arbitrary small $\varepsilon>0$ and for $n>n_{0}(\varepsilon)$ there is a $\varsigma_{v}$-system satisfying (2.5) such that

$$
\max _{\substack{2 \leqq \nu \\ \nu \text { integer }}}\left|s_{\nu}\right| \leqq e^{-\left(\boldsymbol{\alpha}_{0}-\varepsilon\right) u} \text {. }
$$

Hence, if for each integer $n \geqq 1$ and $m \geqq 0$ the estimation (2.6) is valid, then indeed.

$$
\left(\frac{n}{A_{1}(1+n)}\right)^{n} \leqq e^{\left(-\alpha_{0}+\varepsilon\right) n}, \quad \text { i. e. } \quad A_{1} \geqq e^{\alpha_{0}} \sim 1,321,
$$

10. We are going to prove (3.4). Obviously we need only to show

$$
A_{3} \geqq \frac{4}{x} \text {. }
$$

We choose e. g. $n$ big odd, $m=n^{3}$ and with a slight modification of idea of P. STEIN ${ }^{i}$

(10. 1) $\quad z_{j}=e^{\frac{\pi i}{2(m+n)}(2 j-n-1)}, \quad b_{j}=\frac{1}{2^{n-1}}\left(\begin{array}{l}n-1 \\ j-1\end{array}\right) e^{\frac{\pi i n}{4(n+n)}(2 j-n-1)} \quad(j=1,2, \ldots, n)$.

Then we get

$$
\begin{gathered}
f_{0}(y)=\sum_{j=1}^{n} b_{j} z_{j}^{y}=\frac{1}{2^{n-1}} \sum_{j=1}^{n}\left(\begin{array}{c}
n-1 \\
j-1
\end{array}\right) e^{\frac{n i}{2(m+n+n)}(2 j-n-1)\left(y+\frac{n}{2}\right)}=- \\
=\left(\frac{e^{\frac{\pi i}{2(m+n)}\left(y+\frac{n}{2}\right)}+e^{-\frac{\pi i}{2(m+1)}\left(y+\frac{n}{2}\right)}}{2}\right)^{n-1}=\cos ^{n-1}\left\{\frac{x}{2(m+n)}\left(y+\frac{n}{2}\right)\right\} .
\end{gathered}
$$

Let us observe that for all sufficiently large $n$ 's

$$
\left|\sum_{j=1}^{n} b_{j}\right|=\cos ^{n-1} \frac{\pi n}{4(m+n)}=\cos ^{n-1} \frac{\pi}{4\left(n^{2}+1\right)}>\cos ^{n-1} \frac{\pi}{4 n^{2}}>\frac{1}{1+\frac{1}{n}} .
$$

Hence for $m \leqq y \leqq m+n$ we obtain

$$
\begin{gathered}
\left|f_{0}(y)\right| \leqq\left(1+\frac{1}{n}\right)\left|\sum_{j=1}^{n} b_{j}\right| \sin ^{n-1} \frac{x n}{4(m+n)}<\left(1+\frac{1}{n}\right)\left(\frac{\pi}{4} \cdot \frac{n}{m+n}\right)^{n-1}\left|\sum_{j=1}^{n} b_{j}\right|= \\
=\left(1+\frac{1}{n}\right) \frac{4}{\pi}\left(n^{2}+1\right)\left(\frac{n}{\frac{4}{\pi}(m+n)}\right)^{n}\left|\sum_{j=1}^{n} b_{j}\right| .
\end{gathered}
$$

Since for an arbitrary small $\varepsilon>0$ we have for $n>n_{0}(\varepsilon)$

we got

$$
\left(1+\frac{1}{n}\right) \frac{4}{\pi}\left(n^{2}+1\right)<\frac{1}{(1-\varepsilon)^{n}}
$$

$$
A_{3} \geqq \frac{4}{\pi}(1-8)
$$

Q.e.d.

T See J. E. Litrlewood, Math. Notes (12), An inequality for a sum of cosines, Journ. of London Math. Soc., 12 (1937), pp. 217-222. 
11. In order to show (4.7)-(4.8) we take

$$
s_{1}=-2 u, \quad s_{2}=2 v^{2} \quad(v \neq 0) .
$$

Then Newton-Girard's formulae give

$$
\begin{gathered}
a_{1}=2 u=v H_{1}\left(\frac{u}{v}\right), \\
2 a_{2}=-s_{2}-a_{1} s_{1}=-2 v^{2}+4 u^{2}=v^{2}\left\{4\left(\frac{u}{u}\right)^{2}-2\right\}=v^{2} H_{2}\left(\frac{u}{v}\right), \\
a_{2}=\frac{v^{2}}{2 !} H_{2}\left(\frac{u}{v}\right),
\end{gathered}
$$

denoting by $H_{r}(y)$ the $\nu^{\text {th }}$ Hermite polynomial defined in (4.9). Suppose we showed already

$$
a_{m}=\frac{v^{m}}{m !} H_{m}\left(\frac{u}{v}\right) \quad(m=1,2, \ldots, k)
$$

for a $k<n$. Then

$$
(k+1) a_{k+1}=-\left(s_{k+1}+a_{1} s_{k}+\cdots+a_{k} s_{1}\right)
$$

and, using (4.7) and (11.2),

$$
\begin{gathered}
(k+1) a_{k+1}=-\left(a_{k-1} s_{2}+a_{k} s_{1}\right)=-\frac{v^{k+1}}{(k-1) !} H_{k-1}\left(\frac{u}{v}\right) 2+\frac{v^{k}}{k !} H_{k}\left(\frac{u}{v}\right) 2 u= \\
=\frac{v^{k+1}}{k !}\left\{-2 k H_{k-1}\left(\frac{u}{v}\right)+2 \frac{u}{v} H_{k}\left(\frac{u}{v}\right)\right\} .
\end{gathered}
$$

But as well known

$$
2 x H_{k}(x)-2 k H_{k \cdot 1}(x)=H_{k+1}(x)
$$

i. e. from (11.3)

$$
a_{k+1}=\frac{v^{k+1}}{(k+1) !} H_{k+1}\left(\frac{u}{v}\right),
$$

what shows that (11.2) is true for $m=1,2, \ldots, n$. Conversely these values $a_{v}$ assure that $s_{3}=s_{4}=\cdots=s_{n}=0$. For $s_{n+1}$ we have

$$
s_{n+1}=-\left(a_{1} s_{n}+\cdots+a_{n} s_{1}\right)=-\left(a_{n-1} s_{2}+a_{n} s_{1}\right) \text {, }
$$

i. e. from (11.1) and (11.2)

or from (11.4)

$$
\begin{aligned}
s_{n+1} & =-\frac{v^{n+1}}{(n-1) !} H_{n-1}\left(\frac{u}{v}\right) 2+\frac{v^{n}}{n !} H_{n}\left(\frac{u}{v}\right) 2 u= \\
& =\frac{v^{n+1}}{n !}\left\{2 \frac{u}{v} H_{n}\left(\frac{u}{v}\right)-2 n H_{n-1}\left(\frac{u}{v}\right)\right\},
\end{aligned}
$$

$$
s_{n+1}=\frac{v^{n+1}}{n !} H_{n+1}\left(\frac{u}{v}\right)
$$


Since $v \neq 0, s_{u+1}=0$ implies $u=\lambda v$ where

$$
H_{n+1}(\lambda)=0 \text {. }
$$

This proves already (4.7)-(4.8).

For the value $s_{n+2}$ we have in our case

$$
s_{n+2}=-\left(a_{1} s_{n+1}+a_{2} s_{n}+\cdots+a_{n} s_{2}\right)=-a_{n} s_{2}=-2 \frac{v^{n+2}}{n !} H_{n}(\lambda),
$$

i. e.

$$
\max _{\nu=3,4, \ldots,(n+2)}\left|s_{\nu}\right|=-\frac{2}{n !}|v|^{n+2}\left|H_{n}(\lambda)\right| .
$$

If the minimal absolute value of the zeros of

$$
\sum_{\nu=0}^{n} \frac{H_{\nu}(\lambda)}{\nu !} z^{r}=0
$$

is denoted by $A_{n}(\lambda)$, then the maximal one of

$$
\sum_{\nu=0}^{n} \frac{H_{\nu}(\lambda)}{\nu !} v^{\nu} z^{n-\nu}
$$

is $\frac{|v|}{A_{n}(\lambda)}$. Hence, if we choose $v=A_{n}(\lambda)$, we obtained a $\left(z_{1}^{*}, \ldots, z_{n}^{*}\right)$-system with

and

$$
\max _{i=1, \ldots, n}\left|z_{j}^{*}\right|=1
$$

$$
\max _{\nu=3,4, \ldots,(n+2)}\left|z_{1}^{*}{ }^{\nu}+\cdots+z_{i}^{*}{ }^{*}\right|=\frac{2}{n !}\left|H_{n}(\lambda)\right| A_{n}(\lambda)^{n+2} .
$$

An asymptotical determination of $A_{n}(\lambda)$ (or even a good upper estimation of it) and a suitable choice of $\lambda$ would probably result a better lower bound for $A_{1}$ in the Theorem.

(Received 29 January 1955)

\section{О НЕКОТОРЫХ НОВЫХ ТЕОРЕМАХ ТЕОРИИ ДИОФАНТОВЫХ ПРИБЛИЖЕНИЙ В ера Т. Шош и П. Т уран (Будапешт)}

$$
\text { (Р е з юм е) }
$$

Второй из авторов настоящей работы во своей недавно вышедшей книге дал целый ряд применений диофантовых неравенств I, II, III, относящихся к различным ветвьям аиализа и аналитической теории чисел. Улучшение әтих неравенств важно и с точки зрения применений. В настоящей работе авторы значительно улучшают теорему II. В одном из самых важных для применений случаев это улучшение состоит в следующем : Пусть $\max _{j=1, \ldots, n}\left|z_{j}\right|=1$, и пусть $A$ означает наименьшую числовую постоянную, 
для которой

$$
\max _{n+1 \leqq \nu \leqq m+n}\left|s_{\nu}\right| \equiv \max _{m+1 \leqq \nu \leqq m+n}\left|z_{1}^{\nu}+\cdots+z_{n}^{\nu}\right| \geqq\left(\frac{n}{A(m+n)^{\prime}}\right)^{n}
$$

(v принимает целые значения), при всех целых неотрицательных $m$ и целых. $n$. Тогда

$$
1,321<A<2 e^{1+\frac{4}{e}} .
$$

В связи с улучшением оценки снизу возникает вопрос о всех системах $\left(z_{1}, \ldots, z_{n}\right)$, для которых

a) $s_{2}=s_{3}=\cdots=s_{3}=0$,
b) $s_{3}=s_{4}=\cdots=s_{n+1}=0$.

Легко доказать, что с точностью до растяжения и вращения единственная система $\left(z_{1}, \ldots, z_{n}\right)$, удовлетворяющая условию а) состоит из корней уравнения $\sum_{\nu=0}^{n} \frac{z^{n-\nu}}{\nu !}=0$. Несколько труднее доказать, что - опять с точностью до растяжения и, вращения все системы $\left(z_{1}, \ldots, z_{n}\right)$, удовлетворяющие условию b) состоит из корней $n+1$ уравнений

$$
\sum_{\nu=0}^{n} \frac{H_{\nu}(\lambda)}{\nu !} z^{n-\nu}=0
$$

где $H_{\nu}(y)$ есть многочлен Әрмита степени $v$, а $\lambda$ любой корень уравнения $H_{n+1}(y)=0$. 